\title{
Filigrane
}

Écoutes psychothérapiques

\section{La disparition du père : de l'affaissement du symbolique à l’angoisse du réel}

\section{Pascale Roger}

Volume 23, numéro 1, printemps 2014

URI : https://id.erudit.org/iderudit/1026078ar

DOI : https://doi.org/10.7202/1026078ar

Aller au sommaire du numéro

\section{Éditeur(s)}

Revue Santé mentale au Québec

ISSN

1192-1412 (imprimé)

1911-4656 (numérique)

Découvrir la revue

Citer cet article

Roger, P. (2014). La disparition du père : de l'affaissement du symbolique à l'angoisse du réel. Filigrane, 23(1), 67-82. https://doi.org/10.7202/1026078ar

\section{Résumé de l'article}

La symbolisation est la conséquence d'un fait anthropologique : la paternité n'est pas une certitude spontanée, elle est produite par la pensée, contrairement à la maternité qui s'inscrit dans l'expérience sensible. Le " père de l'Oedipe " n'est donc ni le " bon éducateur " ni le " chef ", mais celui qui prend sens comme père pour l'enfant, et le fait entrer dans le registre de la signification et du langage en y incorporant le désir et la loi. Le déclin de la fonction paternelle ou son absence engendre la production archaïque de " père imaginaire " mais aussi la détresse d'un psychisme orphelin à vif, qui, ne pouvant plus compter que sur lui-même, se trouve meurtri du dedans par la pulsion comme du dehors par le réel. Cet état, associé à l'absence de tiers et à la jouissance sans limite entretenues par le social, conditionne les formes perverses de toute-puissance et de violence, véritables défenses contre la psychose. 


\section{La disparition du père: de l'affaissement du symbolique à l'angoisse du réel}

\section{Pascale Roger}

La symbolisation est la conséquence d'un fait anthropologique: la paternité n'est pas une certitude spontanée, elle est produite par la pensée, contrairement à la maternité qui s'inscrit dans l'expérience sensible. Le "père de l'CEdipe " n'est donc ni le "bon éducateur " ni le "chef ", mais celui qui prend sens comme père pour l'enfant, et le fait entrer dans le registre de la signification et du langage en y incorporant le désir et la loi. Le déclin de la fonction paternelle ou son absence engendre la production archaïque de "père imaginaire " mais aussi la détresse d'un psychisme orphelin à vif, qui, ne pouvant plus compter que sur lui-même, se trouve meurtri du dedans par la pulsion comme du dehors par le réel. Cet état, associé à l'absence de tiers et à la jouissance sans limite entretenues par le social, conditionne les formes perverses de toute-puissance et de violence, véritables défenses contre la psychose.

La lune est-elle habitée, l'astronome le sait avec à peu près autant de fiabilité qu'il sait qui était son père, mais pas aussi assurément qu'il sait qui a été sa mère. Ce fut un grand progrès de la culture lorsque les hommes se résolurent à placer l'interférence à côté du témoignage des sens et à passer du droit maternel au droit paternel.

FrEUD, 1909d, 201

$\mathrm{Si}$, pour avoir un père, il faut la parole d'une femme et le consentement d'un homme, nous avancerons qu'il faut aussi la reconnaissance de la fonction du père par le social.

LeBrun, 2002, 50

\section{La symbolisation: un fait anthropologique}

Pour la théorie psychanalytique, la loi instaure un ordre symbolique, qui préexiste au sujet infantile, et selon lequel il va lui falloir se structurer. Le fondement de cette loi, son socle, est constitué par les interdits de l'inceste et du 
meurtre. Elle est transmise par ce que Jacques Lacan a nommé, en référence aux trois registres ${ }^{1}$ (le symbolique, l'imaginaire, le réel), le "père symbolique » qui doit donc être distingué du «père réel» (le géniteur) et du «père imaginaire ». Le père symbolique possède une fonction structurante en tant qu'il nomme l'interdiction œedipienne et demande à l'enfant de renoncer à la mère. Mélanie Klein souligne l'importance capitale du complexe d'CEdipe dont dépendent les problèmes caractériels de «la déformation légèrement névrotique jusqu'à la déformation criminelle»(Klein, 1989, 212). Jean Bergeret explique que notre époque «avec son atmosphère œdipienne devenue quelque peu raréfiée» appelle plus que jamais la considération des «fondements violents non intégrés sous le primat du génital» et produits «avant la mise en action de la triangulation odipienne» ou lorsque «la triangulation œdipienne se trouve entravée» (Bergeret, 1984, 13).

Le père transmet l'ordre symbolique à son enfant grâce au processus de pensée fondé sur le langage. Mais ce processus ne peut avoir lieu sans une élaboration psychique fondamentale, qui est liée à l'incertitude même de la paternité. En effet, ce doute va provoquer un questionnement inaugural débouchant sur la construction intellectuelle du père dans la psyché de l'enfant. Cette symbolisation ${ }^{2}$ portée par le père, nommée «fonction paternelle » par la psychanalyse, ne provient d'aucune position politique ou religieuse (défense du patriarcat) ou d'aucune autorité supérieure par principe ou par choix. Elle doit être entendue comme la conséquence d'une donnée anthropologique: la paternité n'est pas une certitude charnelle et spontanée, elle demande à être appréhendée par la pensée et le langage alors que la maternité s'inscrit d'abord dans l'expérience sensible. C'est pourquoi, il ne faut pas confondre l'ordre anthropologique (la fonction ${ }^{3}$ du père ou la «fonction paternelle») avec l'ordre éducatif (le «rôle du père» dans la famille et la société) et l'ordre social ou politique (la «fonction patriarcale»). Le "père symbolique », le "père de l'Edipe», n'est donc ni le «bon éducateur» ni le «chef», mais il est celui qui prend sens comme père pour l'enfant, et de ce fait l'introduit dans le registre de la signification et du langage en y incorporant le désir et la loi. À notre époque où il est infiniment plus facile, grâce aux tests génétiques, d'acquérir une certitude sur sa paternité, celle-ci reste évidemment le produit d'une démarche volontariste et intellectuelle. Si la paternité moderne est sortie définitivement de la sphère de l'hypothétique pour entrer, à l'instar de la maternité, dans celle de l'irrécusable, elle n'en devient pas pour autant une connaissance charnelle liée à une perception immédiate. Elle reste de toute façon à produire. C'est précisément cette non-immédiateté, cette 
absence de «savoir» naturel et fusionnel chez l'enfant qui, pour Freud, fonde l'importance de l'ordre intellectuel et spirituel:

[...] ce tournant effectué de la mère vers le père désigne en outre une victoire de la spiritualité sur la sensorialité, donc un progrès culturel, car la maternité est attestée par le témoignage des sens, alors que la paternité est une hypothèse édifiée sur une déduction et un présupposé. Le parti pris d'élever le processus de pensée au-dessus de la perception sensorielle s'avère être un pas lourd de conséquences. (Freud, 1939a, 192)

L'établissement du registre symbolique, comprenant la pensée, le langage et la loi (de nature anthropologique et non politique) transmis par le père, étant capital pour la culture - sans être infaillible comme en témoigne l'existence des névroses et des psychoses - la question est alors de savoir ce que devient «l'efficacité symbolique» (Lévi-Strauss, 1958, 223) si la fonction paternelle s'affaiblit ou disparait, ou si elle tend à ne plus se distinguer de la fonction maternelle. Notre propos n'est pas de faire l'historique ou de discuter dans le détail de la transformation de l'imago paternelle et du déclin du patriarcat, provoqués à la fois par les mœurs et la juridiction, et déjà mis en évidence par Freud, Lacan et plusieurs contemporains ${ }^{4}$, mais d'en montrer les conséquences sur le plan symbolique. Que devient l'inscription de la fonction paternelle dans l'inconscient lorsque le géniteur est absent ou défaillant? Quelles sont les conséquences psychiques et sociales d'un affaiblissement, voire d'une disparition de la fonction paternelle? Est-ce qu'une autre entité que le père peut amener à une symbolisation aussi prégnante que celle produite par celui-ci? Si l'existence et la présence du père ne garantissent sûrement pas le travail de symbolisation, comme le montre en général la psychanalyse, et spécifiquement le cas de l'homme aux loups, il n'est en revanche pas possible d'en déduire ipso facto la totale autonomie du «père symbolique » par rapport au «père réel » et donc la remise en question du «père réel » comme agent le plus évident de la castration symbolique. Concernant le père, la disjonction entre réel, imaginaire et symbolique, bien qu'utile sur un plan théorique, s'avère problématique dans la réalité du sujet parce que non dénuée d'effets cliniques.

\section{L'absence de père: "père imaginaire " et angoisse du réel}

Dans l'article «Le déclin du père », Markos Zafiropoulos défend l'idée selon laquelle la thèse de l'affaiblissement des structures familiales et de l'imago paternelle qui «soutient aujourd'hui encore nombre de démarches 
psychanalytiques comme bon nombre d'élaborations sur le postmodernisme [...]» (Zafiropoulos, 2002, 12-13), est le signe d'une nostalgie du père (Vatersensucht), d'un appel au père, entraînant une "orthopédie du père » et « relançant la face la plus morbide du divin ». Il critique alors ce qu' il nomme «appel au père»:

[...] Le danger socio-clinique de l'appel au père réside très exactement dans le retour forcené de la bonasse paternelle assurant la paix pour les fidèles de la même église, mais aussi et du même mouvement les massacres de masse des fidèles de toutes les autres religions. (Zafiropoulos, 2003, 163)

Alors que la thèse de l'affaiblissement des structures familiales peut être analysée à l'opposé comme une justification d'une société sans père devenu un nouvel idéal puissant, il faut interroger la pertinence de cette analyse. Car de quel père et de quel appel s'agit-il ici? L'article de Markos Zafiropoulos produit précisément une confusion. Tout en insistant sur l'inscription subjective de la fonction paternelle, l'article fait paradoxalement un amalgame fâcheux. Il faut distinguer plusieurs appels: celui qui est inconscient et qui concerne notamment le "père imaginaire ", et un autre d'ordre intellectuel qui émerge face à une transformation sociale et en questionne les conséquences psychiques et sociales. Le premier peut déboucher sur une organisation religieuse ou politique possiblement tyrannique, alors que le deuxième, se nourrissant d'une observation lucide, en constitue précisément le meilleur rempart. Ainsi, la reconnaissance psychanalytique et sociale de l'importance du père odipien, qui plus est dans un contexte qui tend à évacuer celui-ci, ne devrait pas être assimilée à une demande de père inconsciente alimentant le «père imaginaire». Cette assimilation ressemble d'ailleurs moins à une réflexion scientifique qu'à une psychanalyse sauvage du social cherchant peut-être à masquer des positions politiques :

La théorie du vidage du père comme de la fonction symbolique dans nos sociétés relève plus de la nostalgie du père et donc du symptôme névrotique que d'une découverte scientifique. (Zafiropoulos, 2007, 1-2)

Par ailleurs, cette affirmation qui attribue au corps social, par le biais de théories qu'il produit, un symptôme, et sous-entend donc un «inconscient collectif», ne peut entrer en correspondance avec la théorie freudienne. En effet, si celle-ci s'intéresse à la dimension collective ou sociale de l'incons- 
cient ainsi qu'aux prédispositions inconscientes du lien social ou de l'institution, elle ne le fait pas en utilisant cette entité autonome qu'est le concept jungien d' «inconscient collectif». Quoi qu'il en soit, l'émergence au plan de la conscience n'est-il pas le meilleur chemin pour stopper une trajectoire souterraine et possiblement pathogène? L'expression " appel au père », que l'auteur de l'article sort de son contexte, ne semble donc pas la plus appropriée pour décrire une réflexion lucide et rationnelle ou des théories sociales en rapport avec des transformations profondes de la société qui montre une disparition du père œdipien. En revanche, au niveau inconscient, il n'est plus à démontrer que le déclin de la fonction paternelle laisse le champ libre à la production inconsciente massive de "père imaginaire ${ }^{5}$ ». Plus le père est absent ou défaillant, plus la demande d'un substitut paternel est forte, liée à des images fantasmatiques et attachée à l'autoritarisme ou à une position de domination. La disparition du père réel peut donc entraîner non seulement une perte ou une absence de symbolique mais surtout l'envahissement d'un père fantasmé qui dans la réalité possède le visage du maître (ou d'un dieu), du chef dont l'autorité, voire la tyrannie, peut être recherchée comme palliatif, ou même projetée dans une dynamique paranoïaque. Cette recherche d'un père imaginaire, véritable contenu d'un roman familial, survient également au moment même où l'enfant découvre que son père n'est pas aussi parfait qu'il le pensait, par exemple en le comparant à d'autres hommes de la société. C'est donc là que réside précisément le véritable «appel au père» (Vatersehnsucht) névrotique, tel qu'il a été formulé par Freud: appel au père qui naît précisément d'un manque ou d'une frustration et se nourrit de toutes les formes de "père imaginaire», du meilleur au plus cruel, mais qui a peu à voir avec le père symbolique ou odipien.

Dans cette configuration, toutes les dérives sont possibles tant au niveau psychique qu'au niveau politique. Car le déclin du père ne produit pas seulement un affaissement (ou une disparition) du symbolique mais bien, face à l'anarchie pulsionnelle, l'activation réactionnelle d'un surmoi exigeant, voire particulièrement cruel et tyrannique, quand un père imaginaire féroce prend la place du père réel et du père symbolique. Quand le symbolique défaille, le manque et le trop convergent, à l'image du père féroce et du père humilié qui se font miroir, ceci illustrant l'apport de Freud concernant la symétrie du surmoi et du ça dans leur cruauté ${ }^{6}$. C'est pourquoi, le déni ou la non-reconnaissance de la disparition de la fonction paternelle, et donc l'absence de symbolisation et de verbalisation de cette réalité, entraîne un «appel au père » archaïque fondé sur une nostalgie trompeuse et angoissée. Cet appel 
souvent inconscient, accompagne l'anarchie pulsionnelle et produit une résurgence surmoïque démesurée sur le plan psychique, qui trouve une prolongation dans le caractère autoritaire, fasciste ou totalitaire dans la sphère collective et politique. Dans son roman autobiographique Ramon, Dominique Fernandez (2010, 64-54, 89, 90, 109) montre comment son propre père Ramon, un intellectuel de gauche, est devenu communiste puis fasciste et collaborateur adhérant aux thèses nazies, par un transfert dans sa vie politique des manques provenant de son éducation familiale qu'il qualifie de «despotisme maternel». Tout au long du roman, il ne cesse de mettre en évidence cette quête du père inassouvie, conséquence directe du régime matriarcal dans lequel Ramon a été élevé, de la défaillance de son propre père à assumer sa fonction paternelle, puis de la perte de celui-ci qui le laissa orphelin. Malgré l'autorité maternelle, aucun ordre symbolique ne semble pouvoir s'élaborer dans la psyché de Ramon qui passe sa vie à rechercher le chef et le guide dans ce qu'ils possèdent de plus autoritaire.

Ainsi, il n'y a aucun danger à une réflexion consciente sur la disparition de la fonction paternelle et «le retour forcené de la bonasse paternelle» ne peut pas en être la conséquence, bien au contraire. Par ailleurs en transmettant le symbolique, le "père réel» permet aussi un contournement puissant de l'angoisse du réel et une régulation psychique. En jouant à la fois le rôle de tampon et de dérivatif, il dispense au sujet d'être meurtri par le réel, comme c'est le cas dans la psychose, et il évite au psychisme l'envahissement pulsionnel névrotique. L'angoisse de castration a donc paradoxalement une fonction de protection contre l'angoisse de mort. Dans sa mise en rapport des étapes de développement de l'humanité et des régressions névrotiques, Freud explique que le réel est premier et précède la relation au père qui viendra finalement dans un deuxième temps « divertir» l'homme de l'horreur du réel, c'est-à-dire détourner de l'angoisse du réel en transformant celle-ci en angoisse de castration:

Il est possible que la nature égoïstement jalouse et sans égards que nous attribuons, d'après des considérations de la psychologie des peuples, au père originaire de la horde humaine, n'ait pas été présente dès le début, mais soit façonnée au cours des pénibles temps glaciaires, comme résultat de l'adaptation à la rigueur. (Freud, 1915, 295)

Ajoutons que pour Freud ${ }^{7}$, l'angoisse de mort et l'angoisse de castration procèdent d'un mécanisme analogique qui est le sentiment d'abandon de la 
part d'une instance protectrice (Dieu, le père, le surmoi). Le surmoi assure donc la protection de l'appareil psychique des dangers externes comme internes, comme en témoignent les deux étapes de sa construction : le dépassement de l'angoisse du réel lors de l'identification au père par idéalisation, puis la métabolisation de la cruauté originaire (amoralisme de la poussée pulsionnelle, ambivalence et désir de meurtre). Là encore, une réflexion sur le déclin du père permet de reconnaître, à l'origine de cet "appel au père » archaïque, certes le manque de père réel (ou le déclin de sa fonction), mais surtout la souffrance insupportable (accentuée d'ailleurs par l'athéisme), la détresse (Hilflosigkeit) d'un psychisme orphelin qui ne peut plus compter que sur lui-même, qui tel des chairs à vif voit son enveloppe déchirée ou manquante et se trouve à la fois douloureusement meurtri du dedans par la pulsion comme du dehors par le réel. Ainsi, quand l'angoisse de castration, liée au père réel (voir Lacan, Séminaire VII, 355) ne trouve plus matière à s'alimenter, c'est la détresse initiale et l'angoisse du réel lui-même (ou angoisse de morcellement dans la psychose) qui prennent le relais: le tiers étant absent, le sujet, abandonné autant par son père ("père réel») que par le surmoi ( «père symbolique»), se trouve désormais seul et écartelé entre le monde et la profondeur abyssale de sa psyché. Ce qui est en jeu avec le "père réel», ce n'est donc pas les limites véhiculées par la loi (interdictions) ou l'imaginaire (restrictions, frustrations) mais tout simplement celles posées par le réel qui touchent à l'existence même et donc à la non-existence, c'est-à-dire à la mort, l'inatteignable, le non-sens et l'irreprésentable, dont la pulsion de mort est le représentant métapsychologique et pulsionnel.

\section{La présence d'un père ou un père en chair et en os}

Bien que les lois de l'ordre symbolique soient plus larges que les lois de la famille, le père en chair et en os (leiblichen Vater), malgré toutes ses imperfections, malgré la névrose, mais surtout grâce à l'incertitude même de sa nature, semble donc le support le plus opérant du Nom-du-père et du processus de symbolisation. Car quand le père est absent, le questionnement sur la paternité change absolument de nature. L'incertitude fondatrice, de part et d'autre entre le père et l'enfant, qui agit symboliquement est remplacée par le questionnement identitaire et narcissique fondamental de l'enfant concernant la filiation: «D’où je viens? Qui je suis? Pourquoi je suis là?» Le chemin vers la symbolisation se trouve alors entravé, voire gravement perturbé, par cette interrogation incessante de nature angoissée parce que fondée sur une absence et une négation. La question : «Où est mon père? » empêche la 
question fondatrice du symbolique — «Cet homme, qui s'occupe de moi ou qui prétend être mon père, est-il bien mon père?» — d'émerger avec toutes ses implications intellectuelles et œdipiennes. Le passage de l'ordre sensoriel à l'ordre intellectuel qui donne accès à la loi et au champ culturel, est mis à mal par l'envahissement de l'imaginaire et de l'angoisse. Comment l'inscription de la fonction paternelle dans la psyché pourrait-elle se produire sans sujet, dans la plus grande abstraction, ou par un transfert sur une entité qui n'est pas de sexe masculin? Il faut bien qu'il y ait un homme devant soi, pour mettre en doute, confirmer ou infirmer, la paternité, processus biologique, et donc produire la médiation indispensable entre le sujet et l'ordre symbolique. Quant à la mère, il lui est tout à fait possible d'endosser le «rôle du père ", c'est-à-dire par exemple d'exercer une autorité éducative efficiente ou de pratiquer avec son enfant des activités ordinairement masculines. Cependant, de par sa nature biologique, elle est incapable de porter cette «fonction paternelle», cette symbolisation. Le réel de la mère ne sera jamais le réel du père (et vice versa), et la mère ne peut incarner du tiers dans l'un qu'elle forme avec l'enfant, et ceci quelles que soient les décisions culturelles ou politiques qui déterminent le contexte dans lequel elle vit. Ce n'est donc pas parce que la fonction du père est une fonction qui n'est pas immédiate, qui est toujours à produire, même pour le père biologique, bref que le père n'est pas une essence ${ }^{8}$, qu'il faut en déduire que le processus de symbolisation peut être initié de façon identique par n'importe quel substitut. Pour que ce principe de tensions ait lieu, il lui faut ses deux pôles: l'enfant et son père.

Le père absent, la fonction paternelle ne pourra se produire qu'à l'aide de prothèses symboliques, la prothèse la plus efficiente étant amenée par une personne de sexe masculin, qui, sans être le père biologique, endosse volontairement le rôle de père et reconnaît l'enfant comme le sien. Ainsi le père adoptif peut instaurer pendant un certain temps une symbolisation parce qu'en toute logique, ce père de substitution aurait pu tout aussi bien être le père biologique; il en a en tout cas la capacité naturelle. Cependant quand le questionnement de l'enfant sur ses origines surgit ou interfère, il est aussi important de verbaliser la carence paternelle pour ne pas mettre en échec ce travail. La découverte que son père adoptif n'est pas son père biologique est aussi productrice de sens pour l'enfant.

Cependant, quand il s'agit d'une institution dans laquelle l'enfant est placé ou qui sert de référent, on doit reconnaitre qu'il n'est pas transmis un ordre symbolique, tel qu'il est défini par la psychanalyse, mais plutôt un ordre éducatif, légal, juridique et réglementaire, ce qui est infiniment plus restrictif, voire 
contradictoire, en tout cas problématique. Car se confronter à la règle de (ou se mettre en règle avec) telle ou telle institution n'est pas synonyme d'accès à un ordre symbolique, mais plutôt l'évitement de la dialectique de l'œedipe, c'est-à-dire du désir et de la loi :

Le règlement prolifère dans les interstices laissés vacants par la loi [...] En contraste de la loi qui édicte ce que doit le sujet, le règlement ainsi conçu édicte ce que le sujet doit vouloir pour être conforme à sa propre puissance. [...] Avec la loi, on n'en a jamais fini, vu qu'elle relance la question de ce que le sujet même désire. L'aubaine du règlement, c'est qu'avec lui «on est quitte», pourvu qu'on s'y conforme. [...] Chaque règlement crée ainsi une zone propre de jouissance, dont les « usagers » sont assurés... à condition de suivre les flèches! [...] Là où la loi laissait un espace entre le manque et la satisfaction, d'où une dialectique pouvait s'embrayer, le règlement ne laisse qu'un tout ou rien, balancement binaire que tranche la lettre du règlement, à la fois fragile et apodictique. [...] de cet Autre, il n'y a absolument rien à espérer, puisqu'il ne nous parle ni ne nous connaît comme sujets. En conséquence, il se réduit littéralement à son mode d'emploi. (Assoun, 1985, 237, 238-239, 240, 241)

Les dimensions inconscientes du règlement ont tout à voir avec la perversité et l'idéalisation de la toute-puissance. Cette modernité qui évacue le père œedipien possède de nombreux exemples dans la société, mais il en existe un assez emblématique. Comme on l'apprend dans un documentaire ${ }^{9}$, des violeurs peuvent se faire rembourser les dédommagements dus à leur victime grâce à la protection civile de l'assurance multirisques habitation de leurs propres parents. Cette absence de véritable réparation par la prise de conscience de l'acte et de la faute par le sujet lui-même, cette disparition de la peine endurée permise par un règlement et de l'argent témoignent parfaitement de la banalisation, voire de la disparition de la notion de culpabilité. La symbolisation n'a pas lieu, le sujet n'a pas accès à la loi. La disparition du symbolique se fait au profit de la conformité au pouvoir et à ce qui est considéré comme la puissance, et laisse toute la place à la jouissance. Dans la société post-moderne et post-judéo-chrétienne, il est possible d'être en règle ou conforme aux prescriptions d'une administration ou d'une institution, et, dans le même temps, de montrer un comportement moralement inacceptable ou cruel. On perçoit de quoi s'alimentent les totalitarismes ainsi que le «despotisme démocratique», tel qu'il a été conceptualisé par Tocqueville, 
comme s'appuyant sur la rationalité. Le terme de «transgression» a désormais moins de sens que ceux de «contravention» ou de «redressement fiscal» compris immédiatement par tout un chacun!

Le père semble donc incontournable au développement psychique, à son inscription dans l'ordre symbolique, à sa structuration identitaire comme aux sentiments de sécurité et de confiance en soi et dans la vie. C'est pourquoi l'encouragement à ce que le père symbolique soit porté par le père réel (ou par un père de substitution), et dans tous les cas par un homme, un père en chair et en os, n'est ni une incitation dogmatique à retourner à un modèle patriarcal ou conservateur, ni un appel à la restauration ou au renforcement de l'autorité, mais bien la reconnaissance de lois anthropologiques fondamentales dont le psychisme et la société semblent ne pas pouvoir se passer sans dégât. Pour entreprendre ce travail de symbolisation l'enfant n'a pas besoin d'une autorité mais d'une présence paternelle: c'est bien là toute la différence... La solution n'est pas d'inscrire ou de privilégier cette fonction paternelle dans la loi, mais de faire en sorte que la loi et les mœurs, portées par les grandes directions politiques et sociales, ne se retrouvent pas en porteà-faux avec cette donnée anthropologique fondamentale, voire qu'elles ne la contredisent pas carrément.

\section{La société sans père: norme, jouissance et toute-puissance}

L'affaissement du symbolique encouragé par l'ordre social possède de nombreuses implications au niveau individuel comme au plan collectif. L'échec de l'école, à la fois par la présence de la violence et de l'agitation extrêmes (les enseignants passent $90 \%$ de leur temps à faire de la discipline ou de la négociation avec les élèves) ainsi que par le recul des savoirs et de l'éducation en général, en est une des manifestations les plus prégnantes: cet échec trahit les profondes modifications qui affectent la condition subjective dans la société contemporaine et l'impossibilité d'agir au nom d'un acquis symbolique implicite et commun. L'appauvrissement du langage, voire sa destruction ${ }^{10}$, et l'insécurité linguistique qui en ressort, sont significatifs de l'affaissement du symbolique autant que de la difficulté de plus en plus grande de vivre, d'agir et de penser ensemble. L'évincement du père a pour effet un contournement du désir, de la culpabilité et de la dette, qui s'accompagne de la dialectique de la toute-puissance et du face-à-face avec le réel dont le déni (du réel, de la filiation, de l'identité sexuelle...) et le clivage sont les moteurs.

Le tiers, l'autre autant que le langage ${ }^{11}$ - celui qui par son existence pose à la fois l'identité du sujet et ses limites, celui par lequel s'établit le lien à l'ori- 
gine de tout projet collectif, celui qui peut sortir le sujet de la problématique perverse caractérisée par le conflit Idéal du moi/réalité - étant en voie de disparition, les configurations de type pervers (ou limite entre névrose et psychose) dans lesquelles la violence, l'égocentrisme et l'exhibition, l'absence ou l'excès d'émotions dominent, rencontrent de moins en moins d'obstacles. Si on s'accorde à dire que «la solution perverse tend à escamoter l'évolution [...] le lent et douloureux processus d'identification au père» (ChasseguetSmirgel, 1999, 6), alors on doit admettre que la disparition du père (réel et symbolique) favorise la condition perverse et l'émergence d'un «monde sans autrui».

La société sans père promet au sujet un surcroît de puissance, et donc de jouissance et d'accomplissement narcissique, à condition que celui-ci accepte de faire une impasse sur son désir et sur ce qui relie celui-ci fondamentalement à la loi. Mais la sensation de puissance et la jouissance, devenues les contreparties de l'aveuglement du sujet sur lui-même, signent finalement et paradoxalement l'aliénation du sujet au collectif: soumission à l'imaginaire et à l'idéal (prédominance de la norme et de la judiciarisation, remplacement de la culpabilité par la honte), dépendance à l'objet assurant la jouissance, constructions identitaires pathologiques (faux-self, personnalité as if, étatslimites) et emprisonnement dans leurs avatars les plus toxiques et mortiferres que sont d'une part le harcèlement moral ${ }^{12}$, l'emprise et la manipulation mentale comme modes de relation perverse à l'autre, et d'autre part la dépression comme mise à nu par l'effondrement des défenses perverses ellesmêmes. N'y a-t-il pas en effet plus fragile qu'un moi, dénué de référence stable et intrapsychique, incapable de sublimer, et en perpétuelle quête de reconnaissance sociale, de confirmation narcissique et de jouissance? Le sujet en dépression se trouve alors à un carrefour décisif où son effondrement psychique semble l'inciter à " choisir » entre la névrose ou la perversion (la première étant en théorie le "négatif» de la seconde), alors que par ailleurs, la société rend la névrose de moins en moins possible précisément par l'affaissement des interdits ou leur changement de nature.

Tout le tragique de la dépression moderne est peut-être là, dans ce gouffre de la psychose qui hante le sujet et contre lequel il se bat inconsciemment. Si au premier abord, on est tenté de penser que le coût de l'appartenance collective pour le sujet, au regard de la satisfaction pulsionnelle, semble avoir diminué, il faut bien reconnaître que le «compromis» amené par le clivage est en réalité extrêmement fragile et absolument trompeur car il ne laisse pas le sujet indemne de souffrances et d'angoisses en recouvrant à peine l'abîme 
de la psychose. Ainsi la conclusion n'est pas satisfaisante: non seulement le coût individuel n'a pas diminué — il a seulement changé de nature —, mais le coût pour la société, quant à lui, a nettement augmenté. On est passé d'une (prétendue) aliénation au père, catalyseur de névroses, à une aliénation sociale ${ }^{13}$ bien réelle. Pour tuer le père, encore faut-il en avoir un! Être vraiment indépendant, se débarrasser du père, ne passe pas par un simple pas de côté, par l'évincement social de celui-ci.

Les malaises de la société contemporaine peuvent être reliés à cet affaissement du symbolique, à ce déni de la castration comme à l'échec de la sublimation qui en est corrélative. Ils témoignent aussi de la prédominance de l'imaginaire et de la jouissance sans médiation:

- Le communautarisme (religieux, ethnique, sexuel, juridique, administratif...) se présente comme un palliatif à la déréliction à l'œuvre et à l'isolement des sujets en recherche de référents dans un monde massifié et globalisé. Cependant il ne fait que mettre en commun un objet de puissante satisfaction.

- Les sectes se construisant sur l'échec de la rationalisation, grâce à l'emprise et à la pensée magique, apportent l'illusion de la protection et du savoir.

- Les pratiques addictives et risquées (aux drogues, à la nourriture, à la consommation, aux jeux, à autrui, au sexe...) dénient le manque et le désir qui se sont transformés en besoins avides inlassablement assouvis par une jouissance sans limite portée par la pulsion de mort.

- La violence morale et physique, trajectoire externe de la pulsion de mort, donne l'illusion du pouvoir et de la maîtrise du réel.

Mais cette idéalisation proprement délirante et mortifère, décrivant autant une "désymbolisation des interdits ${ }^{14}$ » qu'une "passion de la norme ", se nourrissant de dénis autant que de défis souvent inhibiteurs voire absolument écrasants pour le moi (Ehrenberg, 1998, 246, 247, 249), est-elle absolument nouvelle? Si les formes d'organisation sociale contemporaines en rapport avec l'affaiblissement ou la disparition du référent paternel œdipien permettent son plein épanouissement, la métapsychologie freudienne n’a pas oublié d'en poser les fondements par la dissociation des deux fonctions surmoïques «idéal du moi » et «surmoi interdicteur», portant ainsi en germe ce que nous avons théorisé ${ }^{15}$ comme le conflit qui peut caractériser ces deux instances formant un couple, et dont est corrélatif l'échec de la sublimation. De cette dissociation entretenue et exacerbée par le social, que l'on pourrait nommer également disjonction entre le «surmoi induit par le collectif» et le 
«surmoi œdipien», naît chez le sujet un conflit interne, un double bind, qui l'écartèle aux confins de la schizophrénie et caractérise les pathologies les plus contemporaines: d'un côté la loi (le symbolique et les interdits) le plus souvent sous la forme de traces, et de l'autre la norme sociale (forcément relative) s'actualisant dans un idéal collectif qui incite à la jouissance permanente chevillée à la soumission à des règles.

Ainsi le rôle de la psychanalyse n'a jamais été aussi décisif: nommer cette distorsion qui aliène le sujet pour lui réapprendre (ou apprendre) à reconnaître la singularité de son désir, de son histoire et de sa parole, en réarticulant ceux-ci à l'ordre symbolique et au complexe d'CEdipe dont l'universalité pourtant réelle, est paradoxalement remise en cause par la culture et les imaginaires sociaux contemporains. Ainsi, en désignant l'impasse narcissique et la jouissance sociale qui dépersonnalisent le sujet dont la perversité, la paranoïa ou la phobie sont les modes dominants de relation à l'autre, au regard de toutes les tentatives de normalisation et de massification contemporaines, la psychanalyse reste absolument subversive autant que porteuse d'une dimension éthique. Elle propose un retour vers l'humain et ce qui le constitue en propre, la pulsion et le langage lui-même fondé sur la nécessité vitale de faire le lien entre l'individuel et le collectif, alors que la société au cœur même de son progrès, de son organisation politique comme de ses mœurs les plus contemporaines, en appelle à la sortie... espérons non définitive. C'est pourquoi face à ces «nouvelles maladies de l'âme» (Kristeva, 1993, 15-19) qui vident ou mettent à mort l'espace psychique, face aussi à cette «virtualisation du Nom-du-père» devenu inopérant (Lebrun, 2002, 103), la psychanalyse offre un retour à soi qui tient moins de la confirmation narcissique que d'une invitation à la subjectivation et à l'émergence d'une parole libre, et donc à la reconstitution d'une vie intérieure mise à mal par l'affaissement du symbolique encouragé par le champ social.

Pascale Roger japascale.roger@gmail.com

\section{Notes}

1. Le registre symbolique concerne le langage qui ouvre sur la signification, l'élaboration conceptuelle et la loi; celui de l'imaginaire fait référence aux représentations fondées sur les images, aux fantasmes et au narcissisme. Le réel, à distinguer de la réalité, est ce qui existe mais qui n'est pas accessible dans sa totalité par le psychisme: son équivalent psychique est l'inconscient et la pulsion. La référence à ces trois registres n'est pas incontournable pour comprendre le processus de symbolisation et les enjeux du complexe 
d'CEdipe amenés par la théorie freudienne. Néanmoins, elle apporte une certaine clarté dans une réflexion concernant la fonction paternelle.

2. Jacques Lacan rend compte de la psychose à partir d'un échec de la symbolisation qu'il a nommé «forclusion du Nom-du-père». Cette expression est synonyme de déni ou de rejet primordial du "père symbolique» qui transmet la loi et le langage.

3. «1) La fonction. Elle est de l'ordre du langage et de la parole, elle introduit du sens pour un sujet en tant qu'il est structuré par ce que les psychanalystes appellent le complexe d'CEdipe. Elle est ce qui fait sens dans la paternité ou encore, pour reprendre l'expression de C. Lévi-Strauss (1958) à propos de la cure chamanique : elle est de l'ordre d'une "efficacité symbolique”.» (Hurstel, 1989, 244)

4. "En tous ses aspects, la fonction du père subit de véritables mutations. Avec les lois de 1935 (abolition du droit de correction paternel) et surtout de 1970 ("l'autorité parentale" remplace "l'autorité paternelle"), le statut juridique de toute-puissance du père (le "paterfamilias" issu du droit romain) a été abrogé. Avec celles de 1972 (égalité des filiations légitimes et naturelles) la mère a hors mariage l'autorité parentale exclusive.» (Hurstel, 1989, 259)

5. Le cas de «L'homme aux loups » est représentatif: le père de Sergueï Pankejeff n'est pas un «père castrateur», ce sont les femmes de son entourage qui jouent ce rôle, il se montre même extrêmement tendre avec son fils et devient l'objet de son désir. Pour des raisons liées à la séduction qu'il a subie, Sergueï Pankejeff recherche le père symbolique castrateur, un père qui, contrairement à son vrai père, aurait avec lui des rapports punitifs. L'enfant nourrit donc une production massive de "père imaginaire » qui alimente son attitude provocatrice et la recherche de la satisfaction d'être puni par le père.

6. «Du point de vue de la restriction pulsionnelle, de la moralité, on peut dire: le ça est totalement amoral, le moi s'efforce d'être moral, le sur-moi peut devenir hypermoral et alors aussi cruel que seul peut l'être le ça.» (Freud, 1923b, 297).

7. «[...] je m'en tiens fermement à la supposition que l'angoisse de mort doit être conçue comme analogon de l'angoisse de castration, et que la situation à laquelle le moi réagit est le fait d'être délaissé par le sur-moi protecteur — les puissances du destin — par quoi prend fin l'assurance contre tous les dangers. » (Freud, 1926d, 246).

8. "Mais précisément, c'est pour cela que "le père" est tout sauf une essence... Il est le principe de tensions qu'il sert non à résoudre, mais à désigner.» (Assoun, 1989, 48).

9. Claire Barsacq, Nos enfants en danger, Zone interdite, M6, mars 2009.

10. «La peur que les élèves ont des mots reste la traduction d'une démission. L'expression en creux de la fausse identité qu'ils vont tenter d'imposer aux autres et à eux-mêmes, tout en sachant qu'il s'agit d'un leurre. [...] Ceci est grave, car la pose, le non-langage, le vide deviennent rapidement une ontologie. Le costume a le pouvoir de se métamorphoser en chair à une vitesse vertigineuse [...]» (Ladjali, 2007, 23)

11. «[...] il faut, pour être sujet, consentir à un Non! qui semble inscrit dans la spécificité de l'être parlant, puisque du fait de la parole, il s'agit de renoncer à être dans les choses, à être dans l'immédiat. » (Lebrun, 2002, 97)

12. Le harcèlement moral, cette cruauté morale, n'est autre que la forme perverse de l'agressivité naturelle, contenue par la culture et les interdits, définie par Freud comme «le penchant constitutionnel des hommes à s'agresser mutuellement» (Freud, 1930a, 330). Il n'est pas étonnant que ce harcèlement s'épanouisse particulièrement dans la société sans père: « $[. .$.$] de nombreux observateurs, et nous-mêmes dans nos consultations pour vic-$ times de harcèlement moral, avons relevé que ce phénomène apparaissait préférentiellement dans les entreprises et les institutions où l'autorité se trouvait absente, neutralisée, séduite (par le harceleur), divisée, morcelée par des conflits de légitimité ou de rivalité. 
Quand le chat est parti... Quand le responsable n'est pas là, les "petits chefs", les “caïds", les pervers de constitution ou d'occasion "fleurissent". Un des rôles de l'autorité est d'expliciter les conflits, de les organiser et de les arbitrer.» (Prigent, 2002, 509)

13. «Dans le despotisme moderne, l'État-despote est devenu (mortellement) sérieux, ou neutre comme un administrateur, tandis qu'il règne sur une masse de sujets adonnés à leurs plaisirs. [...] C'est parce que les individus sont réduits à l'état d'atomes sensitifs, adonnés à la recherche de leur propre intérêt, que l'État peut régner tranquillement audessus d'eux. Car cet État "aime que les citoyens se réjouissent, pourvu qu'ils ne songent $q u$ 'à se réjouir" (souligné par nous). Autrement dit : "Il travaille volontiers à leur bonheur; mais il veut en être l'unique agent et le seul arbitre" [Tocqueville]. [...] L'eudémonisme systématisé au point que l'individu n'ait plus à assumer ce que son penser comporte de "trouble" et sa vie de "peine", voilà ce que serait en effet le programme totalitaire pleinement réussi.» (Assoun, 1985, 247-248)

14. «Ce besoin patent de l'escalade des défis et de mise à l'épreuve des limites déplace l'axe de lecture du malaise du côté de la perversion. Tout se passe en effet comme si le sujet du "nouveau malaise" s'“ensocialisait" rageusement, selon le mode alterné de la jouissance et du malaise, de l'idéalisation et du déni. Ce que montre le malaise actuel, c’est donc le "clivage" — si patent dans la perversion — avec ses modes de "féchitisation", "jeux transgressifs" qui témoignent d'une "désymbolisation de l'interdit", qui se "dédommage” paradoxalement par une quête de formes inédites de transgression et une sorte de "passion de la norme".» (Assoun, 1993, 158); "Si la dépressivité et la toxicomanie sont devenues les funèbres emblèmes de la (post)modernité, c'est qu'elles désignent ce destin de la pulsion de mort que n'endigue plus un rapport consistant au symbolique.» (Assoun, 1991, 20).

15. Voir «Idéal du moi et surmoi : conflit intrasystémique et échec de la sublimation » (Roger, 2011, 255-278)

\section{Références bibliographiques}

Assoun, P.-L., 1985, Le désir de règlement, désir de légitimation et éthique administrative, in R. Dray (dir.), Psychologie et science administrative, Paris, P.U.F., 230-248.

Assoun, P.-L., 1989, Fonctions freudiennes du père, in M. Augé et al., Le Père: métaphore paternelle et fonctions du père: l'Interdit, la Filiation, la Transmission, Paris, Denoël, 25-50.

Assoun, P.-L, 1991, Malaise de l'idéal et actualité du malaise, Psychologie clinique, nº 6 Paris, Klincksieck, 7-26.

Assoun, P.-L., 1993, Freud et les sciences sociales: psychanalyse et théorie de la culture, Paris, Armand Colin.

Bergeret, J., 1984, La violence fondamentale, l'inépuisable cedipe, Paris, Dunod.

Chasseguet-Smirgel, J., 1999, La maladie d'idéalité: essai psychanalytique sur l'idéal du moi, Paris, L'Harmattan.

Cottet, S., 1989, Freud et le père, in M. Augé et al., Le Père: métaphore paternelle et fonctions du père: l'Interdit, la Filiation, la Transmission, Paris, Denoël, 53-61.

Ehrenberg, A., 1998, La souffrance d'être soi: dépression et société, Paris, Odile Jacob.

Fernandez, D., 2010, Ramon, Paris, Grasset.

Freud, S., 1909d, Remarques sur un cas de névrose de contrainte, OC IX, Paris, P.U.F. 1998.

Freud, S., 1915, Vue d'ensemble des névroses de transfert, OC XIII, Paris, P.U.F, 2005.

Freud, S., 1923b, Le moi et le ça, OC XVI, Paris, P.U.F., 2003.

Freud, S., 1926d, Inhibition, symptôme et angoisse, OC XVII, Paris, P.U.F., 1992.

Freud, S., 1930a, Le malaise dans la culture, OC XVIII, Paris, P.U.F, 1994. 
Freud, S., 1939a, L'Homme Moïse et la religion monothéiste, OC XX, Paris, P.U.F. 2010.

Hurstel, F., 1989, La fonction paternelle, questions de théorie ou: des lois à la Loi, in M. Augé et al., Le Père: métaphore paternelle et fonctions du père: l'Interdit, la Filiation, la Transmission, Paris, Denoël, 235-262.

Klein, M., 1989, Les tendances criminelles chez les enfants normaux, in Essais de psychanalyse, Paris, Payot, 211-228.

Kristeva, J., 1993, Les nouvelles maladies de l'âme, Paris, Fayard.

Lacan, J., 1986, Le séminaire livre VII, L'éthique de la psychanalyse (1959-1960), Paris, Seuil.

Ladjali, C., 2007, Mauvaise langue, Paris, Seuil.

Lebrun, J.-P., 2002, Une nouvelle chance pour le père?, Filigrane, ${ }^{\circ}$ 1, 50-64.

Lebrun, J.-P., 2002, Le sujet post-moderne: psychopathologie des états-limites, Paris, L' Harmattan.

Lebrun, J.-P., 2007, La perversion ordinaire, Paris, Denoël.

Lévi-Strauss, C., 1958, Anthropologie structurale, Paris, Plon.

Prigent, Y., 2002, Expérience d'une consultation spécifique pour victimes de harcèlement moral, Annales médico-psychologiques 160, Paris, Elsevier, 506-511.

Roger, P., 2011, Penser la cruauté aux interactions de la psychanalyse, thèse de doctorat de psychanalyse et psychopathologie fondamentale, Paris VII-Denis Diderot.

Zafiropoulos, M., 2002, L'anthropologie psychanalytique. Lacan durkheimien, in Assoun et Zafiropoulos, L'anthropologie psychanalytique, Paris, Anthropos, 5-15.

Zafiropoulos, M., 2003, Le déclin du père, Topique n ${ }^{8}$ 4, Paris, L’esprit du temps, 161-171.

Zafiropoulos, M., 2007, La fonction symbolique et la théorie du sujet selon Sartre, Lévi-Strauss et Lacan : retour aux sources et actualité, in Assoun et Zafiropoulos, Actualités de la fonction symbolique, Paris, Anthropos, 1-15. 